\title{
DISCUSIONES SOBRE COHESIÓN SOCIAL EN UNIVERSIDADES MEXICANAS: EL CASO DEL SISTEMA DE EVALUACIÓN UNIVECS
}

\author{
Carlos Sancho-Álvarez¹, José González-Such ${ }^{1}$, Jesús Miguel Jornet Meliá1, María Jesús \\ Perales Montolío', Purificación Sánchez-Delgado ${ }^{1}$ y Sonia Ortega Gaite ${ }^{2}$ \\ 1Universidad de Valencia, España, carlos.sancho@uv.es; jose.gonzalez@uv.es; jornet@uv.es; perales@uv.es; \\ purificacion.sanchez@uv.es \\ Universidad de Valladolid, España, sonia.ortega.gaite@uva.es
}

\begin{abstract}
Resumen. Presentamos un estudio realizado en universidades mexicanas a través de la discusión con docentes y estudiantes universitarios sobre el concepto de cohesión social como referente para la evaluación de carreras universitarias, en el marco del proyecto UNIVECS ${ }^{1}$; que tiene la finalidad de aportar evidencias de validación del Modelo de Evaluación, y de su adaptación en México ${ }^{2}$. El objetivo del estudio es conocer la percepción del profesorado y estudiantes sobre su institución en torno a una serie de dimensiones. La metodología se desarrolla a través de 11 grupos de discusión en tres universidades mexicanas. El análisis de contenido se realiza con el programa de análisis cualitativo WebQDA y se presentan los resultados de estudiantes y docentes para una institución. La propuesta de evaluación es capaz de identificar diferentes obstáculos y facilitadores entre las audiencias estudiadas. Se presenta la información más destacada por los participantes para advertir aquellos aspectos más frecuentes o prioritarios. Pretendemos ofrecer una herramienta de análisis y un sistema de evaluación útil para la autoevaluación y diagnóstico institucional, en relación al concepto de Cohesión Social en las carreras universitarias desde un proceso de investigación transformador con y para los agentes implicados.
\end{abstract}

Palabras clave: Cohesión Social; Grupos de Discusión; Universidades Mexicanas; Sistema de Evaluación; Análisis de Contenido; Evaluación de Contexto; Diagnóstico Institucional; Educación Superior.

\section{DISCUSSIONS ABOUT SOCIAL COHESION IN MEXICAN UNIVERSITIES: THE CASE OF THE UNIVECS EVALUATION SYSTEM}

\begin{abstract}
We present a study done in Mexican universities through the discussion with university professors and students about the construct of Social Cohesion as a reference for the evaluation of university degrees, in the background of the UNIVECS Project; the goal is to provide evidences of validation of the Evaluation Model, and of its adaptation in Mexico. The goal of the study is to find out the perception of the teachers and students about their institution in relation to a series of dimensions. The methodology is developed through 9 focus groups in three Mexican universities. The content analysis is done with the qualitative analysis program WebQDA and the results of students and teachers are presented for an institution. The evaluation proposal is capable of identifying different obstacles and facilitators among the audiences studied. It is presented the most outstanding information from the participants to warn those most frequent or priority aspects. We offer an analysis tool and an evaluation system useful for self-evaluation and institutional diagnosis, in relation to the concept of Social Cohesion in the university degrees, from a process of transformative research with and for the agents involved.
\end{abstract}

Keywords: Social Cohesion; Focus Group; Mexican Universities; Evaluation System; Content Analysis; Background Assessment; Institucional Diagnosis; Higher Education.

\footnotetext{
Estudio realizado en el marco del proyecto: UNIVECS "Validación de un sistema de evaluación de titulaciones universitarias basado en un modelo de Cohesión Social" (Ref. EDU2016-78065R); financiado por el Ministerio de Economía y Competitividad de España y apoyado con fondos FEDER de la Unión Europea.

2

"Validación cultural del instrumento de evaluación para la Cohesión Social UNIVECS en universidad mexicanas" (Ref. INV18-01-19-06), financiado por el Subprograma Atracció de Talent 2018 del Vicerrectorado de Investigación de la Universitat de Valéncia; en colaboración con el IISUE-UNAM y gracias al apoyo de la Red Iberoamericana de Investigadores sobre la Evaluación de la Docencia (RIIED).
} 


\section{INTRODUCCIÓN}

Desde el modelo de evaluación educativa hacia la Cohesión Social (CS) (Jornet, 2012) y su consecuente validación en el ámbito escolar (Jornet, Bakieva \& Sánchez-Delgado, 2019), se plantea un lineamiento de investigación en el contexto de educación superior, llamado UNIVECS. El objetivo es identificar cómo se encuentran las instituciones en torno a la CS, así como identificar las aportaciones que realizan las universidades (Jornet \& Bakieva, 2019; González-Such \& Garrido, 2019); también validado en el contexto de diferentes universidades españolas (Jornet, Bakieva \& Sánchez-Delgado, 2020). En este sentido, se trabaja el modelo de evaluación UNIVECS mediante una metodología por comités de expertos para poder identificar las dimensiones del constructo teórico, así como los elementos que pueden aportar a la CS desde las carreras universitarias (González-Such \& Sánchez-Delgado, 2019). Este proceso de juicio de expertos, también llamado comité de expertos o expert panel, es una técnica adecuada para asegurar la validez de contenido del constructo teórico a evaluar (Kitamura \& Kitamura, 2000; Hyrkäs, Appelqvist-Schmidlechner \& Oksa, 2003; Escobar-Pérez \& Cuervo-Martínez, 2008), por ello se ha convertido en muchas áreas de investigación en una importante estrategia (Utkin, 2006).

A partir de todos estos trabajos, surge una nueva línea de estudio sobre el sistema de evaluación para la CS UNIVECS en el contexto mexicano, motivo por el cual se desarrolla otro estudio paralelo para desarrollar un proceso de adaptación contextual desde un planteamiento participativo con docentes y estudiantes universitarios, y poder desarrollar una adecuada sensibilidad cultural para comprender la situación en cada caso de investigación (Perales, Sancho-Álvarez \& González-Such, 2019). Asimismo, se dedica un especial esfuerzo en adecuar el proceso para asegurar la validez cultural en los resultados (Solano-Flores \& Milbourn, 2016), dado que la cultura permea en cualquier aspecto de evaluación (Solano-Flores, Contreras-Niño \& Backhoff, 2006; Wuttke, 2007).

Para ello, debemos tener en cuenta un posicionamiento que integre la situación contextual donde se desarrolla la adaptación (a nivel local de institución), así como a partir de la propia validación resultante avanzar sobre la internacionalización (a nivel global entre países), reafirmando el compromiso de las universidades para la consecución de la CS (Fëdorov, González-Such \& Jornet Meliá, 2013). 


\section{FUNDAMENTO TEÓRICO}

El constructo teórico del modelo de evaluación para la CS UNIVECS se basa en la definición del Consejo de Europa (2005), que atiende a "La capacidad de la sociedad para garantizar la sostenibilidad del bienestar de todos sus miembros, incluido el acceso equitativo a los recursos disponibles, la dignidad en la diversidad y la autonomía personal y colectiva y a la participación responsable" (p. 23).

A su vez, está inserta en su planteamiento la propia definición de CS de la Comisión Económica para América Latina y el Caribe (CEPAL) que hace referencia a "La eficacia de los mecanismos instituidos de inclusión social como a los comportamientos y valoraciones de los sujetos que forman parte de la sociedad" (CEPAL, 2007, p. 19).

Esto implica que la cohesión social en las sociedades modernas no alude a la armonía social resultado de la existencia en un grupo de normas y valores únicos, sino al reconocimiento y valoración de la pluralidad de intereses e identidades existentes. Para ello se apela a un conjunto de valores definidos como universales y que están comprendidos en la dimensión prescriptiva: respeto a la diversidad cultural y a los derechos humanos, solidaridad social, igualdad social, participación cívica responsable para alcanzar metas comunes por medios democráticos, sentido de pertenencia a la Unión Europea, autonomía personal y la democracia como sistema idóneo de organización social (CONEVAL, 2015, p.24).

Para este estudio y desde este concepto de CS intentamos identificar, a partir del modelo UNIVECS, en instituciones mexicanas la contribución de éstas al desarrollo y transformación de la sociedad desde las carreras universitarias, así como realizar un proceso reflexivo de adaptación cultural participativo.

\section{METODOLOGÍA}

A partir del constructo UNIVECS como objeto de análisis en universidades mexicanas, se emplea la técnica de grupo de discusión para generar la reflexión entre el profesorado y el alumnado, entendiendo este método como "una técnica no directiva que tiene por finalidad la producción controlada de un discurso por parte de un grupo de sujetos que son reunidos, durante un espacio de tiempo limitado, a fin de debatir sobre determinado tópico propuesto por el investigador" (Gil, 1993, p. 200-201).

Es importante resaltar que se escoge esta técnica, ya que a diferencia del grupo focal, el grupo de discusión es más abierto e intenta recoger las ideas y percepciones más 
prioritarias y relevantes de los participantes, de forma libre y aleatoria, sin la necesidad de redirigir o motivar la conversación, ya que incluso los momentos de silencio también son significativos en el discurso (Callejo, 2009).

Por ello, se deben reconocer las singularidades que tienen lugar durante y en la conversación, ya que "se trata de establecer y facilitar una discusión y no entrevistar al grupo" (Watts \& Ebbut, 1987, p. 27).

En el estudio participaron tres universidades mexicanas situadas a lo largo del país: Universidad Autónoma de Baja California (UABC) -zona norte-, Universidad Nacional Autónoma de México (UNAM) -zona centro- y la Universidad Autónoma de Yucatán (UADY) -zona sur-; como se puede observar en la figura número 1 a continuación.

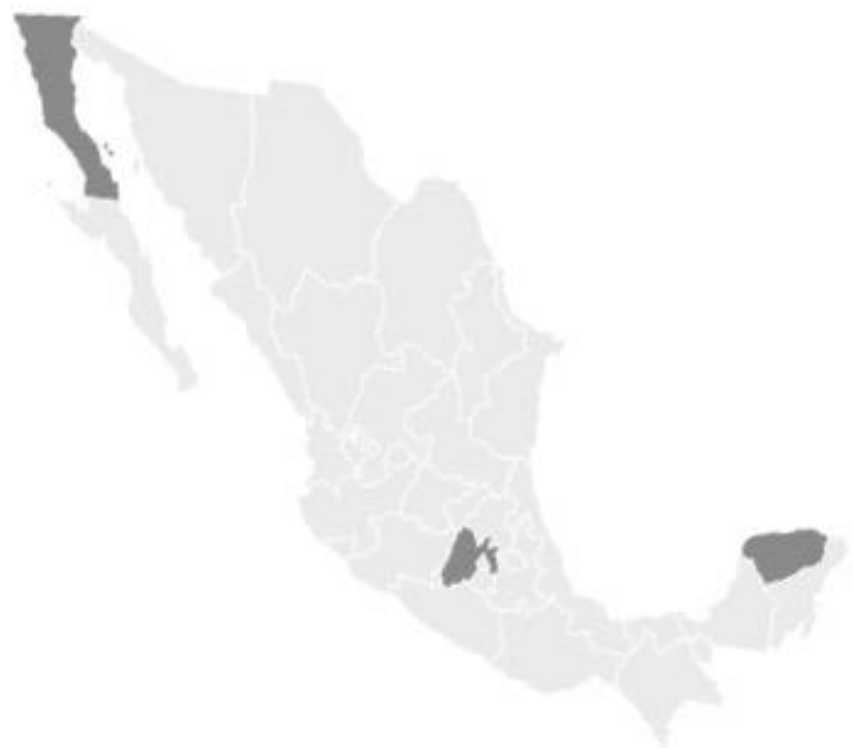

Figura 1. Distribución de grupos de estudio según universidades participantes en México.

En relación a la recomendación de Cohén y Engleberg (1988) y Krueger (1991), se realizaron entre tres y cuatro grupos de discusión por cada universidad, ya que un grupo adicional no suele añadir nueva información en cada zona de estudio.

Asimismo, se desarrollaron un total de 11 grupos entre todas las universidades y audiencias participantes -estudiantes y docentes-, de acuerdo a la pauta de diferentes autores que coinciden en situar el máximo de grupos considerados en un proyecto entre 10 y 12 (Goldman \& McDonald, 1987; Goodman, 1984; Templeton, 1987) -ver tabla 1-. 
Tabla 1. Grupos de estudio por momento de investigación, universidad y audiencia participante.

\begin{tabular}{lllll}
\hline Grupo & Universidad: & Participantes: & Tiempo: & Estado: \\
\hline 1 & UNAM & 12 docentes & $63^{\prime}$ & Ciudad de México \\
2 & UNAM & 10 estudiantes & $74^{\prime}$ & Ciudad de México \\
3 & UNAM & 9 docentes & $62^{\prime}$ & Ciudad de México \\
4 & UNAM & 18 estudiantes & $61^{\prime}$ & Ciudad de México \\
5 & UABC & 12 docentes & $69^{\prime}$ & Baja California \\
6 & UABC & 6 estudiantes & $61^{\prime}$ & Baja California \\
7 & UABC & 8 docentes & $64^{\prime}$ & Baja California \\
8 & UABC & 19 estudiantes & $60^{\prime}$ & Baja California \\
9 & UADY & 6 docentes & $60^{\prime}$ & Yucatán \\
10 & UADY & 11 estudiantes & $69^{\prime}$ & Yucatán \\
11 & UADY & 13 estudiantes & 60 & Yucatán \\
\hline
\end{tabular}

Fuente: Elaboración propia.

Salvo los dos grupos número 4 y 8 , que no se pudieron reducir por problemas de espacios disponibles y para evitar la exclusión de participantes, cada grupo de discusión se realizó con un mínimo 6 participantes y un máximo de 12 en cada momento de investigación, de acuerdo a diferentes autores que consideran estos límites como adecuados para producir un diálogo efectivo (Bers, 1989; Byers \& Wilcox, 1988; Gruning, 1990; Folch-Lyon \& Trost, 1981). Por otro lado, la duración osciló aproximadamente entre 60 y 90 minutos en torno a la adecuación de lbáñez (1986).

Para generar la reflexión y discusión en los grupos, se presentó el constructo UNIVECS con sus definiciones teóricas que surgen a partir de revisión documental y validación por comités de expertos (González-Such \& Sánchez-Delgado, 2019) para cada una de las siguientes dimensiones de medida (citadas en González-Such \& Garrido, 2019, p. 835):

1. Sostenibilidad: "la capacidad de la carrera ${ }^{3}$ universitaria para formar a la comunidad universitaria en competencias que aseguren su desarrollo profesional y respondan a las necesidades presentes y futuras de la sociedad" (Mateu, Garrido y Guaman, 2019).

2. Bienestar Social: "la capacidad de la carrera universitaria para mantener y mejorar la calidad de vida de su comunidad universitaria y de la sociedad, al garantizar y promover el respeto de los Derechos Humanos" (Ancheta, Lázaro y García, 2019).

3. Inclusión: "la capacidad de la carrera universitaria para garantizar la equidad y atender la diversidad de necesidades, capacidades y particularidades de la comunidad universitaria, eliminando las barreras de acceso al aprendizaje, facilitando su participación e incrementando su rendimiento para su desarrollo personal y profesional" (Duart, Marco y Vázquez, 2019).

${ }^{3}$ Las definiciones originalmente contienen en lugar de "carrera" la palabra "titulación", pero en México se presentan así ya que la titulación únicamente se refiere al momento de entrega del título universitario y no a todo el proceso de formación. 
4. Autonomía Personal: "la capacidad de la carrera universitaria para desarrollar entre la comunidad educativa la competencia para decidir y guiar su propio comportamiento" (Serrano, Sanz y Montagud, 2019).

5. Sentido de Pertenencia: "la capacidad de la carrera universitaria para que las personas implicadas en la misma se identifiquen como grupo con sus valores y costumbres, reflejando un compromiso que implique la competencia para participar en decisiones dentro de su comunidad universitaria" (Sánchez-Delgado, Bellver, Moreno, Quintano, Nieto y Ruiz Ruiz, 2019).

6. Confianza: "la capacidad de la carrera universitaria para generar su reconocimiento social a través de la honestidad, la benevolencia y las actitudes y valores compartidos" (Bodoque, Pardo y Martínez, 2019).

7. Movilidad Social: "Ia capacidad de la carrera universitaria para promover y facilitar la mejora del estatus personal, profesional y social de la comunidad universitaria implicada" (Ortega, Perales y Bakieva, 2019).

8. Participación: "la capacidad de la carrera universitaria para desarrollar entre la comunidad universitaria mecanismos formales y no formales de contribución/dinamización de la cohesión social" (Pulido y Alventosa, 2019).

A partir de la visualización de las diferentes dimensiones se plantea la siguiente cuestión para comenzar la discusión: ¿Qué obstáculos y qué facilitadores están dificultando o posibilitando la cohesión social en su carrera universitaria/facultad/institución?

Se solicita la autorización para realizar la grabación de audio en cada grupo de discusión; garantizando la más absoluta confidencialidad de las respuestas en cumplimiento de las Leyes sobre secreto estadístico y protección de datos (CLÁUSULA DE CONFIDENCIALIDAD ACGUV 227/2014 Art. 7.1).

\section{RESULTADOS}

A continuación, se presentan únicamente los resultados para los dos primeros grupos de discusión; el caso 1 con docentes y el caso 2 con estudiantes de la Universidad Nacional Autónoma de México (UNAM) respectivamente, ya que los datos restantes se encuentran en proceso de análisis. Para ello, se realiza un análisis de contenido con el programa WebQDA de la información recogida en función de las diferentes dimensiones de estudio como unidades de análisis (por colores), codificando las aportaciones a partir de la transcripción textual de los grupos y agrupando por factores -ver figura 2-. 


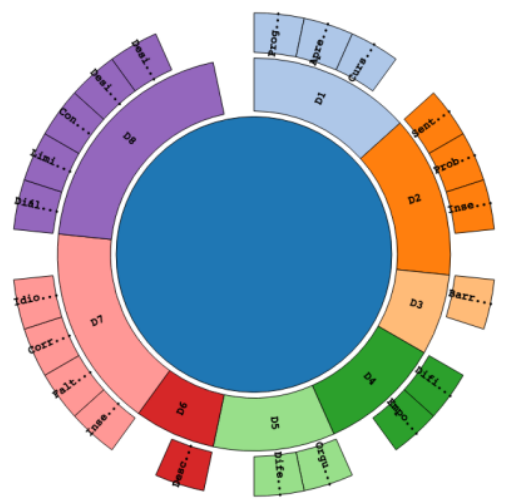

Figura 2. Mapa de densidad de factores y códigos jerárquicos tipo árbol

En la figura 2 podemos observar esquemáticamente cómo se han organizado las aportaciones en en torno a los indicadores de evaluación; de manera más extensa, se irán presentando los códigos completos. El concepto central, o también llamado código origen, sería "Qué obstáculos y facilitadores existen en la carrera/facultad/institución hacia la CS" (zona central de color azul del gráfico), en segundo nivel vienen las unidades de análisis (dimensiones del sistema de evaluación) y, finalmente, el nivel exterior son los sub-códigos en los que se han codificado las respuestas del profesorado y del alumnado participantes.

Tabla 3. Estadísticas de códigos y sub-códigos Fuente: Elaboración Propia

\begin{tabular}{lrr} 
Categorías: & № referencias: & Porcentaje: \\
\hline D1: Sostenibilidad & 15 & 15,5 \\
Programas y asignaturas mejorables & 8 & 8,2 \\
Aprendizaje servicio & 5 & 5,2 \\
Cursos inter-semestrales & 2 & 2,1 \\
D2: Bienestar social & 28 & 28,9 \\
Sentido de justicia & 9 & 9,3 \\
Problemas de violencia & 11 & 11,3 \\
Inseguridad y miedo & 8 & 8,2 \\
D3: Inclusión & 4 & 4,1 \\
Barreras acceso diversidad & 4 & 4,1 \\
D4: Autonomía personal & 9 & 9,3 \\
Dificultad por jerarquías & 3 & 3,1 \\
Empoderamiento estudiantil & 6 & 6,2 \\
D5: Sentido de pertenencia & 10 & 10,3 \\
Orgullo estudiantil & 4 & 4,1 \\
Diferencias por campus & 6 & 6,2 \\
D6: Confianza & 2 & 2,1 \\
Dificultada por injusticas & 2 & 2,1 \\
D7: Movilidad social & 12 & 12,4 \\
Inseguridad social & 2 & 2,1 \\
Falta inserción & 3 & 3,1 \\
Corrupción & 5 & 5,2 \\
Idiomas & Total: & 2,1 \\
D8: Participación & 2 & 17,5 \\
Diálogo social & 3,1 \\
Limitado distancias y horarios & 3 & 5,2 \\
Con comunidades & 5 \\
Desinformación acceso & 5 & 1,2 \\
Desinterés actividades & 5 & 1,0 \\
& 3 & 100,0
\end{tabular}

En todas las dimensiones de análisis se recoge información entre ambas audiencias. 
Sin embargo, se identifican más obstáculos $(62,9 \%)$ que facilitadores $(37,1 \%)$ hacia la CS. En este sentido, se puede destacar la mayor saturación en cuanto a obstáculos en la dimensión 2: Bienestar Social (19,5\%); referidos a "Inseguridad y miedo" (8,2\%) y "Problemas de violencia" (11.3\%). Así como la mayor saturación en cuanto a facilitadores, de manera complementaria en la misma dimensión con un 9,3\% referido a "Sentido de justicia".

Por otro lado, a continuación veremos las aportaciones literales de los participantes referencias- y sus porcentajes textuales. Este soporte permite en función de los resultados codificar las aportaciones en categorías, facilitando la lectura y ordenando la información por porcentajes de respuestas según el tema discutido por los estudiantes y/o docentes.

Se presentan los ejemplos codificados con más frecuencia -mayor porcentaje saturado- en cada dimensión de análisis, presentando dos en caso de empate de datos -ver tabla 3-.

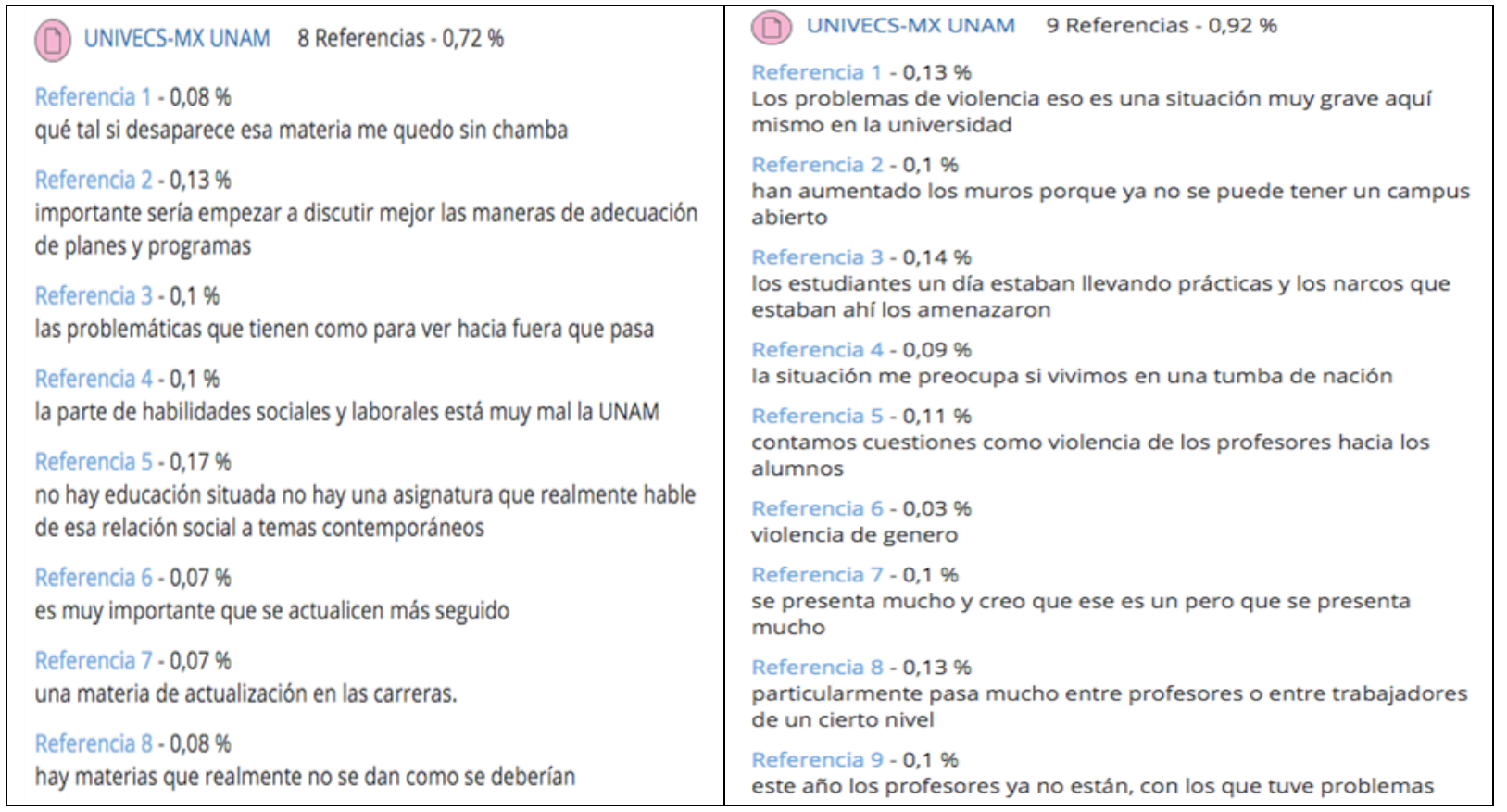

Figura 3. Ejemplo de respuestas para las categorías "Programa y asignaturas mejorables" (D1) y "Problemas de violencia" (D2) 


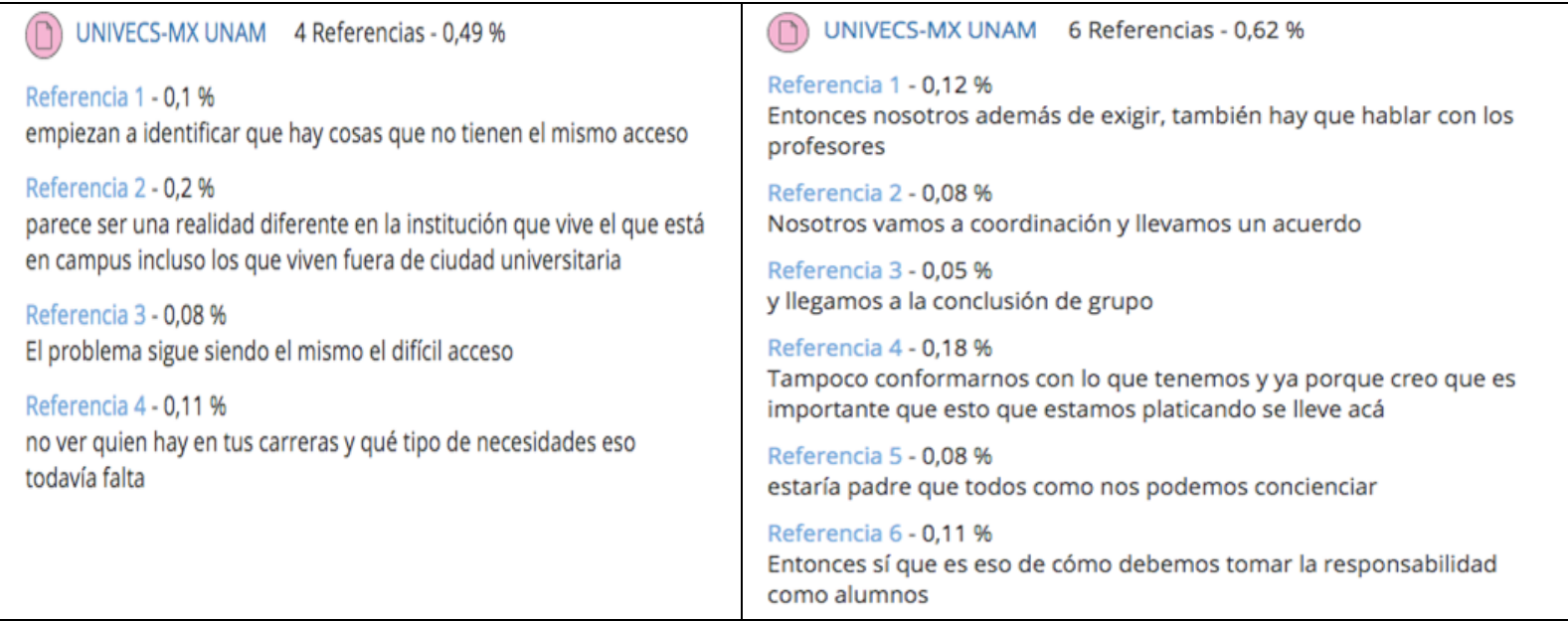

Figura 4. Ejemplo de respuestas para las categorías "Barreras acceso diversidad" (D3) y "Empoderamiento estudiantil" (D4)

\begin{tabular}{|c|c|}
\hline $\begin{array}{l}\text { (1) UNIVECS-MX UNAM } 4 \text { Referencias - 0,44\% } \\
\text { Referencia } 1-0,19 \% \\
\text { por el hecho de haber ingresado y haber recibido su carta de } \\
\text { aceptación en la UNAM ya se sienten orgullosos se sienten parte } \\
\text { Referencia } 2-0,09 \% \\
\text { sí que sabemos que nos sentimos en cuanto a la universidad } \\
\text { Referencia } 3-0,08 \% \\
\text { sentido de pertenencia con respecto a su comunidad } \\
\text { Referencia } 4 \text { - } 0,09 \% \\
\text { yo creo que eso es lo que realmente hace que estén muy unidos }\end{array}$ & $\begin{array}{l}\text { (D) UNIVECS-MX UNAM } 2 \text { Referencias - 0,22 \% } \\
\text { Referencia } 1 \text { - 0,13\% } \\
\text { parece que algunos campus son de segundo nivel y eso afecta } \\
\text { fuertemente a la confianza } \\
\text { Referencia } 2 \text { - 0,09\% } \\
\text { mis reservas en cuanto a la UNAM porque si algo es muy crítico }\end{array}$ \\
\hline
\end{tabular}

Figura 5. Ejemplo de respuestas para las categorías "Orgullo estudiantil" (D5) y "Dificultada por injusticias" (D6)

\section{UNIVECS-MX UNAM 5 Referencias $-0,66 \%$}

Referencia $1 \cdot 0,09 \%$

seguramente conocerás a alguien que te potencian en tu carrera

Referencia $2 \cdot 0,17 \%$

lo que hacen es lo que hacen las iglesias los cambian de capillita y mientras tanto la problemática sigue creciendo

Referencia $3 \cdot 0,1 \%$

otra que me parece sumamente grave es la asignación de contratos

Referencia $4 \cdot 0,1 \%$

Entonces creo que también existe mucho la protección al maestro

Referencia $5 \cdot 0,2 \%$

mismo director quien el no resuelve y el protege a maestros de cosas que él sabe que no se deberian de hacer en la salas de la facultad
UNIVECS-MX UNAM 5 Referencias - 0,53\%

Referencia 1 - 0,07\%

mucho trabajo con las comunidades que le rodean

Referencia 2- 0,08\%

tiene algunas zonas donde lo estudiantes participan

Referencia $3-0,18 \%$

la atención social literalmente en términos de aproximarte a las comunidades yo creo que se alcanza más en las facultades

Referencia 4- 0,08\%

cierta participación social con respecto a la comunidad

Referencia 5 - 0,12\%

discutiendo las problemáticas sociales tendríamos que salir a las comunidades

Figura 6. Ejemplo de respuestas para las categorías "Corrupción" (D7) y "Con comunidades" (D8).

En todas las categorías mostradas se asocian coincidencias -concurrencias- entre estudiantes y docentes, ya que son aportaciones que han identificado ambas audiencias como obstáculos $y / 0$ facilitadores hacia la CS en su carrera/facultad y/o institución universitaria -UNAM, México-. 


\section{CONCLUSIONES}

EI constructo UNIVECS puede permitir evaluar de manera cualitativa la CS en universidades, si se utilizan sus definiciones como detonantes reflexivos y unidades de análisis en grupos de discusión. Por ejemplo, en los casos analizados de UNAM hemos podido comprobar como estos extremos permiten identificar diferentes obstáculos y facilitadores hacia la CS de la institución, a partir de las aportaciones realizadas por los participantes -docentes y estudiantes- en la investigación.

Esta aproximación metodológica se realiza en torno a la interpretación que realiza Sen (2000) sobre el enfoque de capacidades y de acuerdo a la concepción de educación que expone Nussbaum (2007) como "un concepto clave de todas las capacidades humanas" ( $p$. 322). En este sentido, el estudio desarrollado tiene un especial interés por conocer las condiciones de vida y los factores contextuales que posibilitan estas capacidades (Martínez \& París, 2006), entendiendo que estas capacidades pueden estar afectadas por obstáculos y/o facilitadores hacia la cohesión social que condicionan la formación universitaria en cada institución.

Por lo tanto, se establece la posibilidad de mejorar/valorar ciertos factores señalados en la institución analizada, ya que a partir de los resultados se pueden advertir algunas evidencias de facilitadores y obstáculos hacia la cohesión social. En este sentido, se podría establecer una reflexión ante los aspectos señalados con mayores porcentajes, ya que según comentan las propias audiencias: "Los problemas de violencia, eso es una situación muy grave aquí mismo en la universidad" -ver figura 3-; evidencia coincidente con otras investigaciones sobre la presencia de violencia de género en universidades mexicanas (Montesinos \& Carrillo, 2011, Barreto, 2017, Echeverría et al., 2017), sin embargo, no se evidencian otros estudios recientes que traten la inseguridad en la propia universidad.

En efecto, resulta imprescindible seguir conociendo y explorando cada contexto institucional porque únicamente desde las evidencias encontradas podemos mejorar los factores institucionales asociados al proceso educativo universitario. Por ejemplo, en esta línea y de acuerdo a otros antecedentes de investigación en universidades mexicanas, se pudieron generar espacios de reflexión y debate en torno a las condiciones institucionales que estaban incidiendo directamente en la calidad de la formación y en la práctica del aula universitaria (Rueda, Canales, Leyva y Luna, 2014; Luna y Rueda, 2016). 
En este sentido, se continuará analizando el resto de datos de los grupos de discusión participantes para intentar seguir aportando evidencias y devolver la información a los participantes e instituciones colaboradas para realizar la pertinente difusión de resultados, siendo un deber ético en investigación (Sales \& Folkman, 2001).

Algunas limitaciones durante el proceso de investigación han surgido debido a las creencias y concepciones que tiene el profesorado sobre evaluación; algo habitual en estudios de esta índole (Margalef, 2014). El enfoque del sistema de evaluación UNIVECS-MX como herramienta de diagnóstico institucional, en ciertas ocasiones ha podido desvelar algunos entresijos en la institución. Un aspecto que ha podido incomodar y ocasionar algunas resistencias hacia la participación e incluso causar bajas durante la colaboración establecida, ya que son problemáticas habituales en estudios que ofrecen un sistema de participación directa (Balcazar, 2003). Según afirman otros autores, también habitual al evaluar el interior de los centros educativos con la finalidad de mejorarlos (Calatayud, 2009).

Con todo, se continuará la investigación en las instituciones mexicanas participantes realizando diferentes estudios de casos (Stake, 1988) a partir de la aplicación del sistema de evaluación UNIVECS desde una aproximación metodológica complementada -con enfoque cuantitativo y cualitativo-, con el objetivo de comprender mejor la complejidad socioeducativa (Bericat, 1998; Jornet, González-Such y Perales, 2013) y garantizar la máxima validez en los resultados de investigación a través su diseño y construcción junto con los principales implicados (Dellinger y Leech, 2007; Jornet, Perales y González-Such, 2020).

\section{REFERENCIAS}

Balcazar, F. (2003). Investigación acción participativa (iap): aspectos conceptuales y dificultades de implementación. Fundamentos en humanidades, 1-2(7/8), 59-77.

Barreto, M. (2017). Violencia de género y denuncia pública en la universidad. Revista Mexicana de Sociología, 79(2), 261-286.

Bericat, E. (1998). La integración de los métodos cuantitativo y cualitativo en la investigación social. Significado y medida. Barcelona: Ariel.

Bers, T.H. (1989). The popularity and problems of focus-group research. College and University, 64 (3), $260-268$.

Byers, P. \& Wilcox, J. R. (1988): Focus groups: an alternative method of gathering qualitative data in communication research. Comunicación presentada al Annual Meeting of the Speech Communication Association. New Orleans, LA.

Calatayud, M. A. (2009). Entresijos de los centros escolares: develarlos para mejorarlos. Málaga: Aljibe.

Callejo, J. (2009). Introducción a las técnicas de investigación social. Madrid: Editorial Ramón Areces. 
CEPAL. (2007). Un sistema de indicadores para el seguimiento de la cohesión social en América Latina. Santiago de Chile: CEPAL-EUROsociAL.

Cohén, M. C. \& Engleberg, L N. (1989). Focus group research: procedures and pitfalls. Comunicación presentada a la Convention ofthe Eastem Communication Association. Madrid, España.

CONEVAL. (2015). Cohesión social: balance conceptual y propuesta teórico metodológica en México. Ciudad de México: CONEVAL.

Consejo de Europa. (2005). Elaboration concertée des indicateurs de la cohésion sociale - Guide méthodologique. Estrasburgo: Consejo de Europa.

Dellinger, A. \& Leech, N. (2007). Toward a Unified Validation Framework in Mixed Methods Research. Journal of Mixed Methods Research, 14, 309-332.

Echeverría, R., Paredes, L., Kantún, M. D., Batún, J. L. \& Carrillo, C. D. (2017). Acoso y hostigamiento sexual

en estudiantes universitarios: un acercamiento cuantitativo. Enseñanza e Investigación en Psicología, 22(1), 1526.

Escobar-Pérez, J. \& Cuervo-Martínez, A. (2008). Validez de contenido y juicio de expertos: una aproximación a su utilización. Avances en Medición, 6, 27-36.

Fëdorov, A.N., González-Such, J. \& Jornet Meliá, J. M. (2013). Internacionalización de la educación superior y cohesión social: algunos elementos de reflexión (p.161-194). En J. Gacel-Ávila, y N. Orellana, N. (Coord). Educación superior: gestión, innovación e internacionalización. Valencia: JPM Ediciones.

Folch-Lyon, E. \& Trost, J. F. (1981). Conducting focus group sessions. Studies in Family Planning, 12(12),443-449.

Gil, J. (2009). La metodología de investigación mediante grupos de discusión. Enseñanza \& Teaching: Revista Interuniversitaria De DidáCtica, 10, 199-212.

Goldman, A. E. \& Mcdonald, S. S. (1987). The group depth interview. Principies and practica New Jersey: Prentice Hall Inc.

González-Such, J. \& Garrido, P. (2019). Lineamientos para el diseño de estrategias de implementación de la evaluación en el modelo UNIVECS. Comunicación invitada en el XIX Congreso Internacional de Investigación Educativa AIDIPE 2019, Madrid-España.

González-Such, J. \& Sánchez-Delgado, P. (2019). Soluciones metodológicas para la selección y diseño de medidas de percepción para el modelo UNIVECS. Comunicación invitada en el XIX Congreso Internacional de Investigación Educativa AIDIPE 2019, Madrid-España.

Goodman, R. I. (1984). Focus group interviews in media product testing. Educational Technology, August, 39-44.

Grunig, L. A. (1990). Using focus group research in public relations. Public Relations Review, 16(2), 36-49.

Hyrkäs, K., Appelqvist-Schmidlechner, K \& Oksa, L. (2003). Validating an instrument for clinical supervision using an expert panel. International Journal of nursing studies, 40(6), 619 -625.

Ibáñez, J. (1986). Más allá de la sociología. El grupo de discusión: teoría y crítica. Madrid: Siglo XXI Editores.

Jornet, J. M. (2012). Dimensiones Docentes y Cohesión Social: Reflexiones desde la Evaluación. Revista Iberoamericana de Evaluación Educativa, 5(1), 349-362.

Jornet, J. M. \& Bakieva, M. (2019). Diseño de indicadores simples y complejos para la evaluación de entrada, proceso, product y context para el modelo UNIVECS. Comunicación invitada en el XIX Congreso Internacional de Investigación Educativa AIDIPE 2019, Madrid-España.

Jornet, J. M., González-Such, J \& Perales, M. J. (2013). Investigación evaluativa: una perspectiva basada en la complementariedad metodológica. Lima: CREA. 
Jornet, J.M., Bakieva, M. \& Sánchez-Delgado, P. (2019). Una aproximación, mediante juicio, a la validación del modelo de evaluación para el desarrollo de la cohesión social (CS) a partir de la educación. Comunicación invitada en el $8^{\circ}$ Congreso Ibero-Americano en Investigación Cualitativa CIAIQ 2019. Ludomedia: Lisboa, Portugal.

Jornet, J.M., Bakieva, M. \& Sánchez-Delgado, P. (2020) La Cohesión Social como objetivo de la Educación: ¿podemos especificar un modelo de calidad para realizar la evaluación de Sistemas Educativos? Fronteiras: Journal of Social, Technological and Environmental Science (en prensa).

Jornet Meliá, J. M., Perales Montolío, M. J. y González-Such, J. (2020). El concepto de validez de los procesos de evaluación de la docencia. Revista Española de Pedagogía, 78 (276), 233-252. doi: https://doi.org/10.22550/REP78-2-2020-01

Kitamura, T. \& Kitamura, F. (2000). Reliability of clinical judgment of patients' competency to give informed consent: A case vignette study. Psychiatry and Clinical Neurosciences, 54(2), 245-247.

Krueger, R. A. (1991): El grupo de discusión. Guía práctica para la investigación aplicada. Madrid: Pirámide.

Luna, E. \& Rueda, M. (2017). Experiencias de evaluación de la docencia en Iberoamérica. Ensenada: Universidad Autónoma de Baja California.

Margalef García, L. (2014). Formative Aseessment in Higher Education: Teachers' resistance and paradox. Educación XX1, 17 (2), 35-55. doi:10.5944/educxx1.17.2.11478

Martínez, V. \& París, S. (2006). Amartya K. Sen y la globalización. Castellón: Publicaciones de la UJ.

Montesinos, R. \& Carrillo, R. (2011). El crisol de la violencia en las universidades públicas. El Cotidiano,170,49-56.

Nussbaum, M. (2007). Las fronteras de la justicia: consideraciones sobre la exclusión. Madrid: Paidós Ibérica.

Perales, M. J., Sancho-Álvarez, C. \& González-Such, J. (2019). Orientaciones metodológicas para la validación cultural del modelo UNIVECS. Comunicación invitada en el $8^{\circ}$ Congreso Ibero-Americano en Investigación Cualitativa CIAIQ 2019, Lisboa-Portugal.

Rueda, M., Canales, A., Leyva, Y. E., \& Luna, E. (2014). Condiciones contextuales para el desarrollo de la práctica docente. Revista iberoamericana de evaluación educativa, 7(2), 171-183.

Sales, B. D. \& Folkman, S. (2001). Ethics in research with human participants. Washington: APA.

Sen, A. (2000). Desarrollo y libertad. Bogotá: Editorial Planeta.

Solano-Flores, G. \& Milbourn, T. (2016). Capacidad evaluativa, validez cultural y validez consecuencial en PISA. RELIEVE, 22(1), M12. DOI: http://dx.doi.org/10.7203/relieve.22.1.8281

Solano-Flores, G., Contreras-Niño, L. A. \& Backhoff, E. (2006). Traducción y adaptación de pruebas: Lecciones aprendidas y recomendaciones para países participantes en TIMSS, PISA y otras comparaciones internacionales. Revista Electrónica de Investigación Educativa, 8(2).

Stake, R. E. (1988). Investigación con estudio de casos. Madrid: Ediciones Morata.

Templeton, J. F. (1987). Focus groups: a guide for marketing and advertising professionals. Chicago: Probus.

Utkin, L. V. (2006). A method for processing the unreliable expert judgments about parameters of probability distributions. European Journal of Operational Research, 175(1), 385-398.

Watts, M. \& Ebbutt, D. (1987). More than the sum of the parts: research methods in group interviewing. British Educational Research Journal, 13 (1), 25-34.

Wuttke, J. (2007). Uncertainties and bias in PISA. En S. T. Hopmann, G. Brinek, and M. Retzl (Eds.), According to PISA - Does PISA keep what it promises? Berlin: LIT Verlag. 